PROCEEDINGS OF THE

AMERICAN MATHEMATICAL SOCIETY

Volume 132, Number 8, Pages 2197-2204

S 0002-9939(04)07296-X

Article electronically published on March 24, 2004

\title{
ON c-SUPPLEMENTED MAXIMAL AND MINIMAL SUBGROUPS OF SYLOW SUBGROUPS OF FINITE GROUPS
}

\author{
HUAQUAN WEI, YANMING WANG, AND YANGMING LI
}

(Communicated by Stephen D. Smith)

\begin{abstract}
This paper proves: Let $\mathcal{F}$ be a saturated formation containing $\mathcal{U}$. Suppose that $G$ is a group with a normal subgroup $H$ such that $G / H \in \mathcal{F}$.

(1) If all maximal subgroups of any Sylow subgroup of $F^{*}(H)$ are $c$-supplemented in $G$, then $G \in \mathcal{F}$;

(2) If all minimal subgroups and all cyclic subgroups with order 4 of $F^{*}(H)$ are $c$-supplemented in $G$, then $G \in \mathcal{F}$.
\end{abstract}

\section{INTRODUCTION}

All groups considered will be finite. We use conventional notions and notation, as in Huppert [14. $M<\bullet G$ means that $M$ is a maximal subgroup of group $G$.

Maximal and minimal subgroups of Sylow subgroups play an important role in determining the structure of a finite group. They have been studied by many scholars. For instance, Srinivasan [1] showed in 1980 that a finite group $G$ is supersolvable if all maximal subgroups of any Sylow subgroup of $G$ are normal in $G$. Buckley [2] proved in 1970 that a finite group $G$ of odd order is supersolvable if all minimal subgroups of any Sylow subgroup of $G$ are normal in $G$.

Following [1] and [2], extensions were found in [3], 4], [5], [6], [17]. By adding a saturated formation and conditions of complementation and normality, further extensions have been found: [7], 8], 9] for $c$-normality and [10, [11], [12] for $c$ complements. In 1996 Wang generalized the above two results by replacing the normality with the weaker $c$-normality [7]. By minimizing the number of $c$-normal maximal or minimal subgroups, Wei in 2001 extended the results further to a saturated formation containing the class of supersolvable groups [8]. Wang, BallesterBolinches and Guo in 2000 introduced the concept of $c$-supplementation of a finite group which is weaker than both $c$-normality and complementation [10], [11] and generalized the above two results as applications. In [12], Wang, Wei and Li extended the results further to a saturated formation containing the class of supersolvable groups by limiting the $c$-supplementation of maximal or minimal subgroups to the Fitting subgroup of a solvable group.

Received by the editors October 21, 2002 and, in revised form, February 16, 2003.

2000 Mathematics Subject Classification. Primary 20D10, 20 D20.

Key words and phrases. c-supplemented subgroup, supersolvable group, the generalized Fitting subgroup, saturated formation.

Project supported in part by NSF of China, NSF of Guangdong, Fund from Education Ministry of China and ARC of ZSU. 
It is meaningful to remove the solvability of a finite group. Since every simple non-Abelian group $G$ has a trivial Fitting subgroup, one cannot expect a detailed structure if one only gives restrictions on maximal or minimal subgroups of Sylow subgroups of $F(G)$. So the solvability of $G$ with respect to $F(G) \neq 1$ is usually assumed, as in [8], [12, so that the detailed structure of the group can be obtained. However, as we show in the present paper, we can obtain our results on the structure of $G$ if we assume that the maximal or minimal subgroups of the generalized Fitting subgroup of some normal subgroup of $G$ are $c$-supplemented in $G$. The results we mentioned above are special cases of our results, since the generalized Fitting subgroup is a Fitting subgroup when the group is solvable. In fact, we get:

Theorem 1.1. Let $\mathcal{F}$ be a saturated formation containing $\mathcal{U}$. Suppose that $G$ is a group with a normal subgroup $H$ such that $G / H \in \mathcal{F}$. If all maximal subgroups of any Sylow subgroup of $F^{*}(H)$ are c-supplemented in $G$, then $G \in \mathcal{F}$.

Theorem 1.2. Let $\mathcal{F}$ be a saturated formation containing $\mathcal{U}$. Suppose that $G$ is a group with a normal subgroup $H$ such that $G / H \in \mathcal{F}$. If all minimal subgroups and all cyclic subgroups with order 4 of $F^{*}(H)$ are c-supplemented in $G$, then $G \in \mathcal{F}$.

Let $\mathcal{F}$ be a class of groups. We call $\mathcal{F}$ a formation provided that (i) if $G \in \mathcal{F}$ and $N \triangleleft G$, then $G / N \in \mathcal{F}$, and (ii) if $N_{1}, N_{2} \triangleleft G$ such that $G / N_{1}, G / N_{2} \in \mathcal{F}$, then $G /\left(N_{1} \cap N_{2}\right) \in \mathcal{F}$. A formation $\mathcal{F}$ is said to be saturated if $G / \Phi(G) \in \mathcal{F}$ implies that $G \in \mathcal{F}$. In this paper, $\mathcal{U}$ will denote the class of all supersolvable groups. Clearly, $\mathcal{U}$ is a saturated formation (see [10, p. 713, Satz 8.6]). For further discussions of formations, please refer to [13].

\section{Preliminaries}

Definition 2.1 ([10]). A subgroup $H$ of a group $G$ is said to be $c$-supplemented in $G$ if there exists a subgroup $N$ of $G$ such that $G=H N$ and $H \cap N \leq H_{G}=\operatorname{Core}_{G}(H)$. We say that $N$ is a $c$-supplement of $H$ in $G$.

Recall that a subgroup $H$ of $G$ is $c$-normal in $G$ if there exists a normal subgroup $N$ of $G$ such that $G=H N$ and $H \cap N \leq H_{G}$ (7]). Also, a subgroup $H$ of $G$ is complemented in $G$ if there exists a subgroup $N$ of $G$ such that $G=H N$ and $H \cap N=1$. Hence $c$-supplementation is a generalization of $c$-normality and complementation. Two examples in [10] show that $c$-supplementation is more general than both $c$-normality and complementation.

Lemma 2.2 ([10, Lemma 2.1]). Let $G$ be a group. Then

(1) If $H$ is c-supplemented in $G, H \leq K \leq G$, then $H$ is c-supplemented in $K$.

(2) Let $K \triangleleft G$ and $K \leq H \leq G$; then $H$ is c-supplemented in $G$ iff $H / K$ is c-supplemented in $G / K$.

(3) Let $\pi$ be a set of primes, $H$ a $\pi$-subgroup of $G$ and $N$ a normal $\pi^{\prime}$-subgroup of $G$. If $H$ is c-supplemented in $G$, then $H N / N$ is c-supplemented in $G / N$. If, furthermore, $N$ normalizes $H$, then the converse also holds.

(4) Let $H \leq G$ and $L \leq \Phi(H)$. If $L$ is c-supplemented in $G$, then $L \triangleleft G$ and $L \leq \Phi(G)$.

Let $G$ be a group. The generalized Fitting subgroup $F^{*}(G)$ of $G$ is the unique maximal normal quasi-nilpotent subgroup of $G$. Now $F^{*}(G)$ is an important subgroup of $G$ and is a natural generalization of $F(G)$. Its definition and important 
properties can be found in [15, X 13]. We would like to give the following basic facts, which we will use in our proof.

Lemma 2.3. Let $G$ be a group and $N$ a subgroup of $G$.

(1) If $N$ is normal in $G$, then $F^{*}(N) \leq F^{*}(G)$.

(2) $F^{*}(G) \neq 1$ if $G \neq 1$; in fact, $F^{*}(G) / F(G)=\operatorname{Soc}\left(F(G) C_{G}(F(G)) / F(G)\right)$.

(3) $F^{*}\left(F^{*}(G)\right)=F^{*}(G) \geq F(G)$; if $F^{*}(G)$ is soluble, then $F^{*}(G)=F(G)$.

(4) $C_{G}\left(F^{*}(G)\right) \leq F(G)$.

(5) If $K \leq Z(G)$, then $F^{*}(G / K)=F^{*}(G) / K$.

(6) Suppose $P$ is a normal subgroup of $G$ contained in $O_{p}(G)$. Then $F^{*}(G / \Phi(P))$ $=F^{*}(G) / \Phi(P)$.

Proof. (1)-(4) can be found in [15, X 13]. For (5), please refer to [17, Lemma $2.3(4)]$.

(6) Let $\bar{G}=G / \Phi(P)$. Then we get $F^{*}(\bar{G})=F(\bar{G}) E(\bar{G})$, where $E(\bar{G})$ is the layer of $\bar{G}$ (see $[15$, p. 128]. Since $\Phi(P) \leq \Phi(G)$, we have that $F(\bar{G})=\overline{F(G)}$. Let $E / \Phi(P)=E(\bar{G})$. Now $E(\bar{G}) / Z(E(\bar{G}))$ is a direct product of simple nonAbelian groups and $Z(E(\bar{G})) \leq Z(F(\bar{G}))$. So we may think that $E(\bar{G})$ acts on $F(\bar{G})$ the same way as $E(\overline{\bar{G}}) / Z(E(\bar{G}))$ does. Let $L / \Phi(P)=\bar{L}$ such that $\bar{L} Z(E(\bar{G})) / Z(E(\bar{G}))$ is a simple component of $E(\bar{G}) / Z(E(\bar{G}))$ and $Q$ is a Sylow $q$-subgroup of $L$ with $q \neq p$. Since $\bar{L}$ acts trivially on $F(\bar{G})$, we have that $\bar{Q}$ acts trivially on $\bar{P}$. Therefore, $[Q, P] \leq \Phi(P)$. By a well-known application of the Burnside Basis Theorem, we have that $Q$ acts trivially on $P$ itself (16], 7.3.12). For any Sylow $r$-subgroup $R \Phi(P) / \Phi(P)$ of $F(G) / \Phi(P)$ with $r \neq p$, it is clear that $[Q, R] \leq R \cap \Phi(P)=1$. So we have that $Q$ acts trivially on $F(G)$. Note that $\langle\bar{Q} \mid q \neq p\rangle Z(E(\bar{G})) / Z(E(\bar{G}))=\bar{L} Z(E(\bar{G})) / Z(E(\bar{G}))$ by the simplicity of $\bar{L} Z(E(\bar{G})) / Z(E(\bar{G}))$. We have that $[L, F(G)]=1$. Therefore, $[E, F(G)]=1$ and hence $E \leq C_{G}(F(G)) \leq F^{*}(G)$ (see $(2)$ ). Thus $F^{*}(G / \Phi(P)) \leq F^{*}(G) / \Phi(P)$ and so $F^{*}(G / \Phi(P))=F^{*}(G) / \Phi(P)$.

Lemma 2.4 ([10, Theorem 3.3]). Let $G$ be a finite group and $H$ a normal subgroup of $G$ such that $G / H$ is supersolvable. If all maximal subgroups of any Sylow subgroup of $H$ are c-supplemented in $G$, then $G$ is supersolvable.

Lemma 2.5 ([12, Theorem 4.5]). Let $\mathcal{F}$ be a saturated formation containing $\mathcal{U}$. Suppose that $G$ is a group with a solvable normal subgroup $H$ such that $G / H \in \mathcal{F}$. If all maximal subgroups of any Sylow subgroup of $F(H)$ are c-supplemented in $G$, then $G \in \mathcal{F}$.

Lemma 2.6 ([8 Lemma 3]). Let $H$ be a solvable normal subgroup of $G(H \neq 1)$. If every minimal normal subgroup of $G$ that is contained in $H$ is not contained in $\Phi(G)$, then the Fitting subgroup $F(H)$ of $H$ is the direct product of minimal normal subgroups of $G$ that are contained in $H$.

Lemma 2.7 ([12, Theorem 4.1]). Let $\mathcal{F}$ be a saturated formation containing $\mathcal{U}$. Suppose that $G$ is a group with a solvable normal subgroup $H$ such that $G / H \in$ $\mathcal{F}$. If all minimal subgroups and all cyclic subgroups with order 4 of $F(H)$ are c-supplemented in $G$, then $G \in \mathcal{F}$.

Lemma 2.8 (11, Theorem 4.1]). Let $G$ be a group and let $H$ be the supersolvable residual of $G$. If all minimal subgroups and all cyclic subgroups with order 4 of $H$ are c-supplemented in $G$, then $G$ is supersolvable. 
Lemma 2.9 ([9, Theorem 3.2]). Let $G$ be a finite group and $H$ a normal subgroup of $G$ such that $G / H$ is supersolvable. If all minimal subgroups and all cyclic subgroups with order 4 of $F^{*}(H)$ are c-normal in $G$, then $G$ is supersolvable.

Lemma 2.10 ([12, Lemma 2.8]). Let $M$ be a maximal subgroup of $G, P$ a normal p-subgroup of $G$ such that $G=P M$, where $p$ is a prime. Then:

(1) $P \cap M$ is a normal subgroup of $G$.

(2) If $p>2$ and all minimal subgroups of $P$ are normal in $G$, then $M$ has index $p$ in $G$.

\section{Proofs}

\section{The Proof of Theorem 1.1.}

Proof. We consider the following two cases:

Case 1. $\mathcal{F}=\mathcal{U}$.

Suppose that the theorem is false and let $G$ be a counterexample of minimal order. Then we have:

(1) $H=G, F^{*}(G)=F(G) \neq 1$.

By Lemma 2.4, $F^{*}(H)$ is supersolvable. In particular, $F^{*}(H)$ is solvable and so $F^{*}(H)=F(H) \neq 1$ by Lemma 2.3(2), (3). If $H<G$, then the minimality of $G$ implies that $H$ is supersolvable, since $H$ satisfies the hypothesis of the theorem. Then $G$ is supersolvable by Lemma 2.5, a contradiction.

(2) Every proper normal subgroup $N$ of $G$ containing $F^{*}(G)$ is supersolvable.

By Lemma 2.3(1), (3), $F^{*}(G)=F^{*}\left(F^{*}(G)\right) \leq F^{*}(N) \leq F^{*}(G)$; so $F^{*}(N)=$ $F^{*}(G)$. Thus all maximal subgroups of any Sylow subgroup of $F^{*}(N)$ are $c$-supplemented in $N$. Hence $N$ is supersolvable by the minimal choice of $G$.

Now, let $P$ be an arbitrary Sylow $p$-subgroup of $F(G)$. Then

(3) $\Phi(P)=1$, i.e., $P$ is an elementary Abelian $p$-group.

If $\Phi(P) \neq 1$, consider the factor group $G / \Phi(P)$. By Lemma 2.3(5), $F^{*}(G / \Phi(P))$ $=F^{*}(G) / \Phi(P)=F(G) / \Phi(P)$. If $P_{1} / \Phi(P)$ is a maximal subgroup of the Sylow p-subgroup $P / \Phi(P)$ of $F^{*}(G / \Phi(P))$, then $P_{1}$ is a maximal subgroup of $P$. So $P_{1}$ is $c$-supplemented in $G$ by hypothesis. Thus $P_{1} / \Phi(P)$ is $c$-supplemented in $G / \Phi(P)$ by Lemma 2.2(2). If $Q^{*} / \Phi(P)$ is a maximal subgroup of the Sylow $q$-subgroup $Q \Phi(P) / \Phi(P)$ of $F^{*}(G / \Phi(P))$, where $Q$ is a Sylow $q$-subgroup of $F(G)$ and $q \neq p$, then we can denote $Q^{*}=Q_{1} \Phi(P)$, where $Q_{1}$ is a maximal subgroup of $Q$. Now $Q_{1}$ is $c$-supplemented in $G$ by hypothesis, and Lemma $2.2(3)$ implies that $Q^{*} / \Phi(P)$ is $c$-supplemented in $G / \Phi(P)$. In a word, we have proved that all maximal subgroups of any Sylow subgroup of $F^{*}(G / \Phi(P))$ are $c$-supplemented in $G / \Phi(P)$. By the minimality of $G, G / \Phi(P)$ is supersolvable. Since $\Phi(P) \leq \Phi(G), G$ is supersolvable, a contradiction.

(4) There is no subgroup of order $p$ normal in $G$.

If not, let $P_{0}$ be such a subgroup of $G$. Then $P_{0} \leq P$. Since $P_{0} \leq Z(P) \leq$ $Z(F(G)), F(G) \leq C_{G}\left(P_{0}\right) \leq G$. Note that $C_{G}\left(P_{0}\right)$ is normal in $G$, and $F^{*}\left(C_{G}\left(P_{0}\right)\right)$ $=F^{*}(G)=F(G)$. If further $C_{G}\left(P_{0}\right)<G$, then $C_{G}\left(P_{0}\right)$ is supersolvable by (2). Since $G / C_{G}\left(P_{0}\right)$ is cyclic, $G$ is supersolvable by Lemma 2.5 , a contradiction. If $C_{G}\left(P_{0}\right)=G$, then $P_{0} \leq Z(G)$. By applying Lemma $2.3(6), F^{*}\left(G / P_{0}\right)=F^{*}(G) / P_{0}$. With the same argument in (3), we know that all maximal subgroups of any Sylow subgroup of $F^{*}\left(G / P_{0}\right)$ are $c$-supplemented in $G / P_{0}$. The minimal choice of $G$ 
implies that $G / P_{0}$ is supersolvable, and so $G$ is supersolvable by Lemma 2.5 , also a contradiction.

(5) Write $R=P \cap \Phi(G)$. Then $R \neq 1$.

If $R=1$, then by Lemma 2.6, $P=L_{1} \times \cdots \times L_{s}$, where $L_{i}(i=1, \ldots, s)$ are minimal normal in $G$. Let $L_{1}^{*}$ be a maximal subgroup of $L_{1}$. Then $L_{1}^{*} \times$ $L_{2} \times \cdots \times L_{s}=P_{1}$ is a maximal subgroup of $P$. Set $K_{1}=L_{2} \times \cdots \times L_{s}$. Then $\left(P_{1}\right)_{G}=K_{1}$. For if not, then $K_{1}<\left(P_{1}\right)_{G}$, because $K_{1} \leq\left(P_{1}\right)_{G}$. Thus $\left(P_{1}\right)_{G}=$ $\left(P_{1}\right)_{G} \cap L_{1}^{*} K_{1}=K_{1}\left(\left(P_{1}\right)_{G} \cap L_{1}^{*}\right)$ implies that $\left(P_{1}\right)_{G} \cap L_{1}^{*} \neq 1$. Furthermore, $1<$ $\left(P_{1}\right)_{G} \cap L_{1}^{*} \leq\left(P_{1}\right)_{G} \cap L_{1}<L_{1}$, where $\left(P_{1}\right)_{G} \cap L_{1}$ is normal in $G$. This is contrary to the minimal normality of $L_{1}$ in $G$. Hence $\left(P_{1}\right)_{G}=K_{1}$. Since $P_{1}$ is $c$-supplemented in $G$, there exists $N_{1} \leq G$ such that $G=P_{1} N_{1}$ and $P_{1} \cap N_{1} \leq K_{1}$. It follows that $G=P_{1} N_{1}=L_{1}^{*} K_{1} N_{1}$. If further $L_{1}^{*} \cap K_{1} N_{1} \neq 1$, then $1<L_{1} \cap K_{1} N_{1} \leq L_{1}$, where $L_{1} \cap K_{1} N_{1} \triangleleft\left\langle L_{1}, K_{1} N_{1}\right\rangle=G$. By the minimal normality of $L_{1}, L_{1} \cap K_{1} N_{1}=L_{1}$, i.e., $L_{1} \leq K_{1} N_{1}$. Hence $G=K_{1} N_{1}$ and $P_{1}=P_{1} \cap K_{1} N_{1}=K_{1}\left(P_{1} \cap N_{1}\right)=K_{1}$, i.e., $L_{1}^{*}=1$, contradicting $L_{1}^{*} \cap K_{1} N_{1} \neq 1$. Thus $G=L_{1}^{*} K_{1} N_{1}$ with $L_{1}^{*} \cap K_{1} N_{1}=1$, and so $L_{1} \cap K_{1} N_{1}=L_{1}$ is a normal subgroup of $G$ with order $p$. This is contrary to $(4)$.

(6) $F(G)=P$, and $G$ has a unique minimal normal subgroup contained in $R$, say $L$.

Let $Q$ be the Sylow $q$-subgroup of $F(G)$, and let $L$ be minimal normal subgroup of $G$ contained in $R$, where $q \neq p$. Then $Q$ is elementary Abelian by (3). By the properties of a generalized Fitting subgroup (see [15], p. 128), $F^{*}(G / L)=$ $F(G / L) E(G / L)$ and $[F(G / L), E(G / L)]=1$, where $E(G / L)$ is the layer of $G / L$. Since $L \leq \Phi(G), F(G / L)=F(G) / L$. Now set $E / L=E(G / L)$. Since $Q$ is normal in $G$ and $[F(G) / L, E / L]=1,[Q, E] \leq Q \cap L=1$, i.e., $[Q, E]=1$. Therefore, $F(G) E \leq C_{G}(Q)$. If $C_{G}(Q)<G$, then $C_{G}(Q)$ is supersolvable by (2). Thus $E(G / L)=E / L$ is supersolvable. The semisimplicity of $E(G / L) / Z(E(G / L))$ implies that $E(G / L)=Z(E(G / L))$. So $E(G / L) \leq F(G / L)$ and $F^{*}(G / L)=F(G) / L$. With the same argument in (3), we have that $G / L$ satisfies the hypothesis of the theorem. By the minimality of $G, G / L$ is supersolvable and so is $G$, a contradiction. If $C_{G}(Q)=G$, then $Q \leq Z(G)$. By Lemma 2.3(6), $F^{*}(G / Q)=F^{*}(G) / Q=F(G) / Q$. Similarly, $G / Q$ is supersolvable and so is $G$ by Lemma 2.5 , a contradiction.

We know similarly that the minimal normal subgroup of $G$ contained in $R$ is unique.

(7) $P=\left\langle x_{1}\right\rangle \times \cdots \times\left\langle x_{m}\right\rangle \times R$, where $\left\langle x_{i}\right\rangle \neq 1$ and $\left\langle x_{i}\right\rangle R \triangleleft G, i=1, \ldots, m$.

If $R=P$, then by Lemma 2.2(4), all maximal subgroups of $P$ are normal in $G$. This implies that there is a subgroup of order $p$ normal in $G$ since $P$ is elementary Abelian, a contradiction. Hence $R \neq P$. Now $\Phi(G / R)=1$. Then by Lemma 2.6, $P / R=\left(H_{1} / R\right) \times \cdots \times\left(H_{m} / R\right)$, where $H_{i} / R(i=1, \ldots, m)$ are minimal normal in $G / R$. With the same argument as in (5), we know that $H_{i} / R(i=1, \ldots, m)$ are all of order $p$ because all maximal subgroups of $P / R$ are $c$-supplemented in $G / R$. Again, $P$ is an elementary Abelian $p$-group. So $H_{i}$ is of the form $\left\langle x_{i}\right\rangle R, i=1, \ldots, m$.

(8) For any maximal subgroup $R^{*}$ of $R,\left(T R^{*}\right)_{G} \cap R \neq 1$, where $T=\left\langle x_{1}\right\rangle \times \cdots \times$ $\left\langle x_{m}\right\rangle$.

If not, $\left(T R^{*}\right)_{G} \cap R=1$. Then $\left(T R^{*}\right)_{G} \cap\left\langle x_{1}\right\rangle R$ is of order 1 or $p$. Note that $\left(T R^{*}\right)_{G} \cap\left\langle x_{1}\right\rangle R$ is normal in $G$, and its order must not be $p$ by (4). Thus $\left(T R^{*}\right)_{G} \cap$ $\left\langle x_{1}\right\rangle R=1$. Similarly, $\left(T R^{*}\right)_{G} \cap\left\langle x_{1}\right\rangle\left\langle x_{2}\right\rangle R=\cdots=\left(T R^{*}\right)_{G} \cap T R=1$, i.e., $\left(T R^{*}\right)_{G}=$ 1. Since $T R^{*}$ is maximal in $P$, by the hypothesis, there exists $N \leq G$ such that 
$G=\left(T R^{*}\right) N$ with $T R^{*} \cap N=1$. Thus $P \cap N$ is a normal subgroup of $G$ of order $p$, also a contradiction.

(9) Final contradiction.

From (6) and (8), $L \leq\left(T R^{*}\right)_{G} \cap R$ for any maximal subgroup $R^{*}$ of $R$. Now let $N$ be the $c$-supplement of $T R^{*}$ in $G$. Then $\left(T R^{*}\right)_{G} \cap R=T R^{*} \cap\left(T R^{*}\right)_{G} N \cap R=$ $R^{*} \cap\left(T R^{*}\right)_{G} N$. Thus $L \leq \bigcap_{R^{*}<\cdot R}\left(R^{*} \cap\left(T R^{*}\right)_{G} N\right)=\Phi(R) \cap\left(\bigcap_{R^{*}<\cdot R}\left(T R^{*}\right)_{G} N\right)=1$, a final contradiction.

Case 2. $\mathcal{F} \neq \mathcal{U}$.

By case $1, H$ is supersolvable. In particular, $H$ is solvable and so $F^{*}(H)=F(H)$. Hence $G \in \mathcal{F}$ by Lemma 2.5 .

This completes our proof.

\section{The Proof of Theorem 1.2.}

Proof. By Lemma 2.7, we need only prove that $H$ is solvable. Suppose this is false, and let $G$ be a counterexample of minimal order. Then

(1) $F^{*}(H)=F(H) \neq 1$.

In fact, $F^{*}(H)$ is supersolvable by Lemma 2.8. So $F^{*}(H)=F(H) \neq 1$ by Lemma 2.3(2), (3).

(2) $\Phi(H) \neq F(H)$.

If it is not so, then by Lemma 2.2(4), all minimal subgroups and all cyclic subgroups with order 4 of $F^{*}(H)$ are normal in $G$. By Lemma 2.9, $H$ is supersolvable, a contradiction.

(3) For any proper normal subgroup $K$ of $H, K$ is solvable.

Since $K$ is normal in $H, F^{*}(K) \leq F^{*}(H)$ by Lemma 2.3(1). Also, all minimal subgroups and all cyclic subgroups with order 4 of $F^{*}(K)$ are $c$-supplemented in $K$. The minimal choice of $H$ implies that $K$ is solvable.

(4) There exist $O_{p}(H)$ and $M<\bullet H$ such that $O_{p}(H) \cap M<\bullet O_{p}(H)$.

Since $\Phi(H) \neq F(H)$, there exist $O_{p}(H)$ and $M<\bullet H$ such that $O_{p}(H) \nsubseteq \nsubseteq M$. Then $H=O_{p}(H) M$. To show $O_{p}(H) \cap M<\bullet O_{p}(H)$ we consider the following two cases:

(4.1) $p>2$.

If $O_{p}(H)$ has at least one minimal subgroup $\langle x\rangle$ nonnormal in $H$, then by Lemma 2.2(1), $\langle x\rangle$ is $c$-supplemented in $H$; i.e., there is a subgroup $N$ of $H$ such that $H=\langle x\rangle N$ and $\langle x\rangle \cap N=1$. Furthermore, $N$ is a maximal subgroup of $H$ and $O_{p}(H) \cap N$ is a normal subgroup of $H$ by Lemma 2.10(1). Again, $O_{p}(H)=O_{p}(H) \cap$ $\langle x\rangle N=\langle x\rangle\left(O_{p}(H) \cap N\right)$. If $O_{p}(H) \cap N \leq M$, then $H=O_{p}(H) M=\langle x\rangle M$ with $\langle x\rangle \cap M=1$. This implies $\left|O_{p}(H): O_{p}(H) \cap M\right|=|H: M|=|\langle x\rangle|=p$. Hence $O_{p}(H) \cap M<\bullet O_{p}(H)$. If $O_{p}(H) \cap N \nsubseteq M$, then $H=\left(O_{p}(H) \cap N\right) M$, where $x$ is not in $O_{p}(H) \cap N$. With the same argument we may assume that all minimal subgroups of $O_{p}(H) \cap N$ are normal in $H$. By Lemma 2.10(2), $\mid O_{p}(H)$ : $O_{p}(H) \cap M|=| H: M \mid=p$; so $O_{p}(H) \cap M<\bullet O_{p}(H)$.

(4.2) $p=2$.

Let $\pi(H)=\left\{p_{1}, p_{2}, \ldots, p_{n}\right\}$, and let $M_{p_{i}}$ be a Sylow $p_{i}$-subgroup of $M$, where $i=$ $1,2, \ldots, n$ and $p_{1}=2$. Then we know easily that $O_{2}(H) M_{2}=H_{2}$ is a Sylow 2subgroup of $H$. Now, let $P_{1}$ be a maximal subgroup of $H_{2}$ containing $M_{2}$, and set $P_{2}=P_{1} \cap O_{2}(H)$. Then $P_{1}=P_{2} M_{2}$. Moreover, $P_{2} \cap M_{2}=O_{2}(H) \cap M_{2}$. So $\left|O_{2}(H): P_{2}\right|=\left|O_{2}(H) M_{2}: P_{2} M_{2}\right|=\left|H_{2}: P_{1}\right|=2$, i.e., $P_{2}<\bullet O_{2}(H)$. Again, for each $i \neq 1, O_{2}(H) M_{p_{i}}$ is supersolvable by Lemma 2.7, hence $O_{2}(H) M_{p_{i}}=$ 
$O_{2}(H) \times M_{p_{i}}$. Furthermore, $P_{2} M_{p_{i}}$ forms a group, where $i=1,2, \ldots, n$. Hence $P_{2}\left\langle M_{p_{1}}, M_{p_{2}}, \cdots, M_{p_{n}}\right\rangle=P_{2} M$ also forms a group. Since $\left|O_{2}(H): P_{2}\right|=2$ and $P_{2} \cap M=O_{2}(H) \cap M$, it follows that $P_{2} M<O_{2}(H) M=H$. By the maximality of $M$ in $H, P_{2} M=M$ and hence $P_{2} \leq M$. Thus $O_{2}(H) \cap M=P_{2} \cap M=P_{2}$. Therefore, $O_{2}(H) \cap M<\bullet O_{2}(H)$.

(5) Final contradiction.

From (4), $H=O_{p}(H) M$. Set $L=O_{p}(H) \cap M$. Then $L$ is normal in $H$ by Lemma 2.10(1) and $H / L=\left(O_{p}(H) / L\right)(M / L)$. Since $O_{p}(H) / L$ is a normal subgroup of $H / L$ of order $p, F(H) / L \leq C_{H / L}\left(O_{p}(H) / L\right)$. Write $C_{H / L}\left(O_{p}(H) / L\right)=C / L$; then $C$ is normal in $H$ and $F(H) \leq C$. If $C<H$, then $C$ is solvable by (3). Note that $H / C \cong(H / L) /(C / L)$ is cyclic; so $H$ is solvable, a contradiction. If $C=H$, then $O_{p}(H / L) \leq Z(H / L)$. This implies that $O_{p}(H) / L$ centralizes $M / L$; so $M / L$ is a normal subgroup of $H / L$. Thus $M$ is a normal subgroup of $H$. Furthermore, $M$ is solvable by (3). The solvability of $H / M$ implies that $H$ is solvable, a final contradiction.

This completes our proof.

Remarks. Theorems 1.1 and 1.2 are not true for non-saturated formations. Let $\mathcal{F}$ be the formation composed of all groups $G$ such that $G^{\mathcal{U}}$, the supersolvable residual, is elementary Abelian. Clearly $\mathcal{U} \subseteq \mathcal{F}$, but $\mathcal{F}$ is not saturated. Set $G=S L(2,3), H=Z(G)$. Then $G / Z(G)$ is isomorphic to the alternating group of degree four and so $G / H \in \mathcal{F}$. The other hypotheses in Theorems 1.1 and 1.2 are satisfied since $H$ is of order 2 , but $G$ does not belong to $\mathcal{F}$.

\section{ACKNOWLEDGMENT}

The authors would like to thank T. R. Berger for his help. Thanks also to the referee for his/her helpful comments.

\section{REFERENCES}

[1] S. Srinivasan, Two sufficient conditions for supersolvability of finite groups, Israel J. Math. 35(3) (1980), 210-214. MR 81f:20044

[2] J. Buckley, Finite groups whose minimal subgroups are normal, Math. Z. 116 (1970), 15-17. MR 41:6967

[3] M. Asaad, On maximal subgroups of Sylow subgroups of finite groups, Communications in Algebra 26(11) (1998), 3647-3652. MR 99k:20039

[4] M. Assad, M. Ramadan, and A. Shaalan, The influence of $\pi$-quasinormality of maximal subgroups of Sylow subgroups of Fitting subgroups of a finite group, Arch. Math. 56 (1991), 521-527. MR 92d:20022

[5] M. Ramadan, Influence of normality on maximal subgroups of Sylow subgroups of a finite group, Acta Math. Hungar. 59(1-2) (1992), 107-110. MR 93c:20042

[6] A. Ballester-Bolinches and M. C. Pedraza Aguilera, On minimal subgroups of finite groups, Acta Math. Hungar. 73 (1996), 335-342. MR 97j:20019

[7] Y. Wang, $c$-Normality of finite groups and its properties, J. Algebra 180 (1996), 954-965. MR 97b:20020

[8] H. Wei, On $c$-normal maximal and minimal subgroups of Sylow subgroups of finite groups, Comm. Algebra 29(5) (2001), 2193-2200. MR 2002d:20030

[9] Y. Wang, The influence of minimal subgroups on the structure of finite groups, Acta Math. Sinica, English Series 16(1) (2000), 63-70. MR 2001c:20035

[10] Y. Wang, Finite groups with some subgroups of Sylow subgroups $c$-supplemented, J. Algebra 224 (2000), 467-478. MR 2001c:20036 
[11] A. Ballester-Bolinches, Y. Wang, and X. Guo, c-supplemented subgroups of finite groups, Glasgow Math. J. 42 (2000), 383-389. MR 2001k:20031

[12] Y. Wang, H. Wei, and Y. Li, A generalization of a theorem of Kramer and its applications, Bull. Austral. Math. Soc. 65 (2002), 467-475. MR 2003f:20025

[13] K. Doerk and T. O. Hawkes, Finite Soluble Groups, De Gruyter, Berlin, 1992. MR 93k:20033

[14] B. Huppert, Endliche Gruppen I, Springer-Verlag, Berlin and New York, 1967. MR 37:302

[15] B. Huppert and N. Blackburn, Finite Groups III, Springer-Verlag, Berlin and New York, 1982. MR 84i:20001b

[16] W. R. Scott, Group Theory, Prentice-Hall, Inc., Englewood Cliffs, New Jersey, 1964. MR 29:4785

[17] Y. Li and Y. Wang, The influence of minimal subgroups on the structure of a finite group, Proc. Amer. Math. Soc. 131(2) (2003), 337-341. MR 2003h:20032

Department of Mathematics, Zhongshan University, Guangzhou 510275, China and Department of Mathematics, Guangxi Teacher's College, Nanning, 530001, China

E-mail address: weihuaquan@163.com

Lingnan College and Department of Mathematics, Zhongshan University, Guangzhou, 510275, CHINA

E-mail address: stswym@zsu.edu.cn

Department of Mathematics, Guangdong College of Education, Guangzhou, 510310, CHINA

E-mail address: liyangming@gdei.edu.cn 